\title{
Spectroscopic estimate of surface gravity for a planetary member in the $\sigma$ Orionis cluster
}

\author{
E. L. Martín ${ }^{1}$ \\ Institute of Astronomy. University of Hawaii at Manoa. 2680 Woodlawn Drive, Honolulu, \\ HI 96822, USA \\ M. R. Zapatero Osorio ${ }^{2}$ \\ LAEFF-INTA, Villafranca del Castillo, PO Box 50727, E-28080 Madrid, Spain
}

\begin{abstract}
We present intermediate-resolution $(\mathrm{R}=1500)$ near-infrared spectroscopy from $1.17 \mu \mathrm{m}$ to $1.37 \mu \mathrm{m}$ of the spectral type $\mathrm{T}$ planetary candidate member in the $\sigma$ Orionis cluster SOri 70 reported by Zapatero Osorio et al. (2002). The new data have been obtained with NIRSPEC at the Keck II telescope. The best fit of our mid-resolution spectrum of S Ori 70 with theoretical spectra gives $\log \mathrm{g}=3.5 \pm 0.5 \mathrm{~cm} \mathrm{~s}^{-2}$ and $\mathrm{T}_{\text {eff }}=1,100_{-100}^{+200} \mathrm{~K}$. The low gravity of this object derived from spectral synthesis supports its youth and membership to the young $\sigma$ Orionis cluster. Using evolutionary models for an age of $3 \mathrm{Myr}$, we obtain a mass of $3 M_{\text {Jup }}$ and a radius of $0.16 R_{\odot}$, independent of the distance to the object. Our analysis confirms that S Ori 70 is the lowest mass cluster planet so far identified in the galaxy.
\end{abstract}

Subject headings: techniques: spectroscopic, open clusters and associations: individual ( $\sigma$ Orionis) — stars: low-mass, brown dwarfs — stars: mass function stars: formation - planetary systems: formation

\section{Introduction}

Objects with masses below the substellar mass limit $\left(M<0.072 M_{\odot}\right)$ fail to stabilize on the main-sequence and cool down to very low temperatures. Their observational study has started only recently, but it is progressing vigorously. A focus point in the research about substellar mass objects (SMOs) is the minimum mass that free-floating objects in young clusters and associations can have. Particularly, it has been suggested that the faintest and 
coolest objects discovered through deep imaging in Orion have masses below the deuteriumburning limit at $0.013 M_{\odot}$ or $\sim 14 M_{\text {Jup }}$, where $1 M_{\odot}=1047 M_{\text {Jup }}$ (Lucas \& Roche 2000; Zapatero Osorio et al. 2000; Lucas et al. 2001). Such mass limit has been used in the literature to distinguish between brown dwarfs (BDs) and planets (Burrows et al. 1997). Another mass limit that has been discussed lies at $4 M_{\mathrm{Jup}}$, where the interior equation of state changes from being dominated by electron degeneracy pressure to having a significant contribution by metallic hydrogen (Liebert's contribution in Boss et al. 2003). In this paper we adopt the D-burning limit as the dividing line between BDs and planets, so that we are consistent with our previous work in this cluster.

SMOs have also been found orbiting main-sequence stars. The mass distribution of companions to low-mass main-sequence stars within 3 AU shows that SMOs with masses between 70 and $20 M_{\mathrm{Jup}}$ are rare, but their frequency increases below about $10 M_{\mathrm{Jup}}$ (Queloz, Santos and Mayor 2002). On the other hand, there are indications that BDs may be frequent at large separations from stars (Gizis et al. 2000; Potter et al. 2002). Theoretical work has shown that it is possible that planetary mass companions to stars form by gravitational collapse rather than by agglomeration of planetesimals (Boss 2001; Bate, Bonnell \& Bromm 2003). This scenario would seem more plausible if there were also free-floating planets that have directly formed from the collapse and fragmentation of molecular clouds, or that have been ejected by interactions with stars and other SMOs.

The $\sigma$ Orionis cluster is a prime location to search for SMOs because of its young age $(\sim 3 \mathrm{Myr})$, distance $(\sim 440 \mathrm{pc})$ and low reddening (Sherry 2003). A cluster of X-ray emitting

young stars was discovered by Walter, Wolk \& Sherry (1998). A sequence of SMOs has been found extending to masses below the D-burning limit (Zapatero Osorio et al. 2000; Béjar et al. 2001). The coolest and faintest object so far detected in any young cluster or association is S Ori 70 (Zapatero Osorio et al. 2002). In this paper we report spectroscopic observations of S Ori 70 that have more than a factor of 10 higher spectral resolution than our previous data. We use the higher resolution spectrum to improve previous estimates of the gravity and temperature by fitting with synthetic spectra. We confirm that S Ori 70 has a low gravity, which strongly supports its membership in the $\sigma$ Orionis cluster.

\section{Observations and data reduction}

On 2002 November 24th, we observed S Ori J053810.1-023626 (hereafter S Ori 70) with the Keck II near-infrared spectrometer NIRSPEC (McLean et al. 1998). Meteorological conditions were slightly non-photometric (occasional light cirruses) and the seeing ranged from 0.6 arcsec to 0.9 arcsec, as measured in the NIRSPEC images. 
Total exposure times were $4200 \mathrm{~s}$ and $600 \mathrm{~s}$ for the $J$-band spectra of S Ori $70(\mathrm{~J}=20.28$, Zapatero Osorio et al. 2002) and 2MASS J0559191-140449, respectively. The latter object is a relatively bright T5 dwarf $(\mathrm{J}=13.82$, Burgasser et al. 2002). The observing strategy employed for S Ori 70 was as follows: two individual integrations of $600 \mathrm{~s}$ at three different positions along the entrance slit separated by about $13^{\prime \prime}$. An additional exposure of $600 \mathrm{~s}$ was obtained at one of the positions. Only two single integrations of $300 \mathrm{~s}$ were collected for 2MASS J0559191-140449. In order to remove telluric absorptions due to the terrestrial atmosphere, the near-infrared featureless A0V-type stars HD 15820 and HD 63817 were observed very close in airmass (typically within 0.05 airmasses). White light calibration images were taken at the beginning and at the end of the observing night.

Raw data were reduced within $\mathrm{IRAF}^{1}$ and following standard techniques in the nearinfrared. Nodded images were subtracted to remove the sky background and dark current. The object spectra were then optimally extracted using subroutines of the TwODSPEC package. The extracted spectra were divided by their corresponding normalized extracted flatfields. Wavelength calibration $(r m s=3 \AA$ ) was performed using $33 \mathrm{OH}$ sky emission lines. The hydrogen $\mathrm{P} \beta$ absorption line at $1.2818 \mu \mathrm{m}$ in the spectra of the A0V-type stars was interpolated before the corresponding science spectra were divided by them to cancel terrestrial features. To complete the data reduction, we multiplied the spectra of our targets by the black body spectrum for the temperature of $9480 \mathrm{~K}$, which corresponds to the A0V class (Allen 2000).

Our final NIRSPEC spectrum of S Ori 70 is shown in Figure 1. It has a resolving power of $\mathrm{R}=1500$. We also show the spectrum of 2MASS J0559191-140449 obtained the same night. The shape of the spectra are different, indicating different physical conditions in the photosphere. Lucas et al. (2001) showed that triangular shapes in near-infrared spectra of L dwarfs could be explained by the low gravity of SMOs in the Trapezium. In the next section we analyze these conditions using theoretical tools. We find that the triangular shape of the J-band spectrum of S Ori 70 is caused by a surface gravity lower than that of 2MASS J0559191-140449, consistent with having a larger radius as expected for a younger object.

\footnotetext{
${ }^{1}$ IRAF is distributed by National Optical Astronomy Observatory, which is operated by the Association of Universities for Research in Astronomy, Inc., under contract with the National Science Foundation.
} 


\section{Scientific analysis}

In our analysis we use synthetic spectra for T dwarfs that have been developed by Allard et al. (2001). They include the total condensation of dust grains below the photosphere (COND models) and the latest AMES list of $\mathrm{H}_{2} \mathrm{O}$ lines (Partridge \& Schwenke 1997). These models reproduce well the blue near-infrared colors of $\mathrm{T}$ dwarfs in the $\sigma$ Orionis cluster (Martín et al. 2001; Zapatero Osorio et al. 2002). In Figure 2, we show the effects of different surface gravities in the synthetic spectra calculated with COND atmospheric models. A triangular shape is expected to develop for lower gravities due to enhanced methane and steam absorption. The strength of the KI doublet at 1.25 microns is also greater for lower gravities.

Our NIRSPEC spectrum of S Ori 70 has been compared with a grid of theoretical spectra using a least square minimization technique. The best fit is shown in Figure 3. It was obtained for a COND model with $T_{\text {eff }}=1100 \mathrm{~K}$ and $\log g=3.5 \mathrm{~cm} \mathrm{~s}^{-2}$. We estimate that the internal $1 \sigma$ uncertainty of our fitting technique is ${ }_{-100}^{+200} \mathrm{~K}$ and $\pm 0.5 \mathrm{~cm} \mathrm{~s}^{-2}$, respectively.

Using the spectroscopically derived surface gravity and temperature, we can place S Ori 70 in an evolutionary diagram that distance independent (Figure 4). The object lies on the 3 Myr isochrone for a mass of 3 Jupiters using the evolutionary models of Burrows et al. (1997). A similar result was obtained from the models of Chabrier et al. (2000). Higher mass, older T dwarfs, have higher gravities, such as those inferred for the T dwarfs Gl $229 \mathrm{~B}$ and G1570 D, also shown in Figure 4. If S Ori 70 were a brown dwarf in the line of sight to the $\sigma$ Orionis cluster, it should have a higher gravity. The low gravity of this object implies a very young age that is fully consistent with the cluster's age (Béjar et al. 2001; Oliveira et al. 2002; Sherry 2003).

For very young ages $(\leq 1 \mathrm{Myr})$, the ages and masses derived from evolutionary models may depend strongly on the initial conditions assumed in the calculations (Baraffe et al. 2002). However, at an age of $3 \mathrm{Myr}$, the models used to obtain the age and mass of S Ori 70 are thought to be safely independent of the initial conditions.

\section{Discussion}

Spectroscopic analysis of gravity sensitive features has been recognized as a powerful tool to estimate the masses of SMOs (Davidge \& Boeshaar 1991). As they contract with time, the gravity of SMOs changes by more than two orders of magnitude. Very young SMOs have low gravities (log g 3.0), while old ones have high gravities (log g 5.5). The strengths of the molecular absorption bands increase with decreasing surface gravity because 
of changes in the temperature structure of the atmosphere. At lower gravity a larger column abundance of molecules is required to compress the atmosphere to a given pressure. Spectral synthesis analysis of observed spectra have confirmed that lower gravity objects with late-M and L spectral types have stronger molecular bands (Allard et al. 2001; Basri et al. 2000; Gorlova et al. 2003; Leggett et al. 2001; Lucas et al. 2001; Mohanty et al. 2003; Schweitzer et al. 2001, 2002). As shown in Figure 2, gravity effects are also significant in the spectrum of $\mathrm{T}$ dwarfs.

S Ori 70 is currently the coolest object identified in any cluster (Zapatero Osorio et al. 2002). Consequently, it deserves particular attention because it indicates that there could be a numerous population of planetary members of clusters. The estimated mass of S Ori 70 overlaps with the masses of planetary companions to stars, giving an impetus to the ideas that giant planets form by rapid gravitational processes that happen in molecular cloud cores, disks, and filaments (Boss 2001; Bate, Bonnell \& Bromm 2003).

The angular distance of S Ori 70 from the naked-eye O9.5V star $\sigma$ Ori (HR 1931) is 8'.65, corresponding to a projected separation of $182676 \mathrm{AU}$ at $352 \mathrm{pc}$. This star is actually a quintuple system where the most distant companion is a B2 star at 42 ". The total mass of these 5 stars is about $60 \mathrm{M}_{\odot}$. The maximum separation of binaries with O-type primaries is about $110000 \mathrm{AU}$ (Abt 1986). Thus, the separation from the star $\sigma$ Ori to the planetary object S Ori 70 is of the same order of magnitude as the widest binaries with O-type primaries.

Could S Ori 70 be part of a planetary system surrounding the massive multiple stellar system?. Circumbinary disks are know to exist around low-mass stars (e.g., Potter et al. 2000). However, it is not known whether very large disk structures could exist around highmass multiple stars. A dusty proto-planetary disk without a central star has recently been discovered at only 1200 AU from $\sigma$ Ori (van Loon \& Oliveira 2003), suggesting that planetary formation is still ongoing in this region. However, the typical separation between stars in the $\sigma$ Orionis cluster is about 50000 AU. In fact, there are 3 brown dwarfs and one star within 65000 AU of S Ori 70. We conclude that S Ori 70 is likely a planetary member in the cluster, but not particularly bound to any star or brown dwarf. Could S Ori 70 have been ejected from an unstable multiple stellar or planetary system?. We do not have kinematic information so we cannot constrain this hypothesis. Nevertheless, we note that the escape velocity from the cluster $(\mathrm{Vesc}=0.65 \mathrm{~km} / \mathrm{s})$ is lower than the typical ejection velocities produced in the simulations of Bate et al. (2002). Most ejections occur very early in the evolution of the unstable systems (age $<0.1 \mathrm{Myr}$ ), so it is unlikely that S Ori 70 has been recently ejected.

The presence of planetary members in open clusters that are not bound to any particular star opens a new perspective to the diversity of planets in the universe. A matter of ongoing debate is the most suitable nomenclature for planetary objects that are not orbiting solar- 
type stars. We adhere to a wide use of the term "planet" that includes all objects that do not develop nuclear reactions in their cores, independently of where they may be found or how they formed. Qualifiers may be added to specify the environmental conditions, such as "pulsar planets", "solar-system planets", and "close-in planets" which have already been used in the literature. We propose to call planets such as S Ori 70, which are located in a star cluster but do not orbit any particular star, "cluster planets". Our proposition differs from the definition of the IAU Working Group on Extrasolar Planets (Boss et al. 2003), which states that: "Free-floating objects in young star clusters with masses below the limiting mass for thermonuclear fusion of deuterium are not planets, but are sub-brown dwarfs (or whatever name is most appropriate)."

Cluster planets are probably numerous in the galaxy as suggested by the IMF presented in Béjar et al. (2001). The $\sigma$ Orionis cluster is probably not bound because it does not have enough mass (Sherry 2003). Thus, cluster planets become "free-floating planets" after several million years. Free-floating planets are likely to populate the solar neighborhood.

We thank France Allard for sending us synthetic spectra, and David Barrado y Navascués, Victor Sánchez Béjar and Mark Marley for comments that helped to improve the manuscript. MRZ was supported by the Ramón y Cajal postdoctoral program. This material is based upon work supported by the National Science Foundation under Grant No. 0205862. Any

opinions, findings, and conclusions or recommendations expressed in this material are those of the author(s) and do not necessarily reflect the views of the National Science Foundation. The authors wish to extend special thanks to those of Hawaiian ancestry on whose sacred mountain of Mauna Kea we are privileged to be guests. Without their generous hospitality, the Keck II telescope observations presented therein would not have been possible.

\section{REFERENCES}

Abt, H. A. 1986, ApJ, 304, 688

Allard, F., Hauschildt, P. H., Alexander, D. R., Baraffe, I., \& Chabrier, G. 1996, ApJ, 465, L123

Allard, F., Hauschildt, P. H., Alexander, D. R., Tamanai, A., \& Schweitzer, A. 2001, ApJ, 556,357

Allen, C. W., in Allen's Astrophysical Quantities, Fourth Edition, ed. Arthur N. Cox, New York: Springer Verlag, 151 
Baraffe, I., Chabrier, G., Allard, F. \& Hauschildt, P. H. 2002, A\&A, 382, 563

Basri, G. et al. 2000, ApJ, 538, 363

Bate, M. R., Bonnell, I. \& Bromm, V. 2003, MNRAS, 339, 577

Béjar, V. J. S. et al. 2001, ApJ, 556, 830

Boss, A. P. 2001, ApJ, 551, L167

Boss, A. P., Basri, G., Kumar, S. S., Liebert, J., Martín, E. L., Reipurth, B. \& Zinnecker, H. 2003, in IAU Symposium 211: Brown Dwarfs, E. L. Martín (ed), 529

Boss, A. P. et al. 2003, in IAU Symposium 211: Brown Dwarfs, E. L. Martín (ed), 529

Burgasser, A. et al. 2002, ApJ, 564, 421

Burrows, A. et al. 1997, ApJ, 491, 856

Chabrier, G., Baraffe, I., Allard, F. , \& Hauschildt, P. H. 2000, ApJ, 542, 464

Davidge, T. J. \& Boeshaar, P. C. 1991, AJ, 102, 267

Geballe, T. R., Saumon, D., Leggett, S. K., Knapp, G. R., Marley, M. S., \& Lodders, K. 2001, ApJ, 556, 373

Gizis, J. E. et al. 2001, ApJ, 551, L163

Gorlova, N., Meyer, M. R., Liebert, J., \& Rieke, G. H. 2003, ApJ, in press

Leggett, S. K., Allard, F., Geballe, T. R., Hauschildt, P. H., \& Schweitzer, A. 2001, ApJ, 548, 908

Lucas, P. W. \& Roche, P.F. 2000, MNRAS, 314, 858

Lucas, P. W., Roche, P. F., Allard, F., \& Hauschildt, P. H. 2001, MNRAS, 326, 695

Martín, E. L., Zapatero Osorio, M. R., Barrado y Navascués, D., Béjar, V. J. S. \& Rebolo, R. 2001, ApJ, 558, L117

McLean, I. S. et al. 1998, Proc. SPIE, 3354, 566

Mohanty, S., Basri, G., Jayawardhana, R., Allard, F., Hauschildt, P., \& Ardila, D. 2003, ApJ, submitted

Partridge, P. \& Schwenke, 1997, J. Chem Phys., 106, 4618 
Potter, D. E., Close, L. M., Roddier, F., Roddier, C., Graves, J. E., \& Northcott, M. 2000, ApJ, 540, 422

Potter, D. E. et al. 2002, ApJ, 567, L133

Queloz, D., Santos, N. C., \& Mayor, M. 2002 in Proceedings of the First Eddington Workshop on Stellar Structure and Habitable Planet Finding, B. Battrick, F. Favata, I. W. Roxburgh \& D. Galadi (eds), ESA SP-485, Noordwijk: ESA Publications Division, 117

Oliveira, J. M., Jeffries, R. D., Kenyon, M. J., Thompson, S. A., \& Naylor, T. 2002, A\&A, 382, L22

Schweitzer, A., Gizis, J. E., Hauschildt, P. H., Allard, F., \& Reid, I. N., 2001, ApJ, 555, 368

Schweitzer, A., Gizis, J. E., Hauschildt, P. H., Allard, F., Howard, E. M., \& Kirkpatrick, J. D., 2002, ApJ, 566, 435

Sherry, W. H. 2003, PhD Thesis, State University of New York at Stony Brook

van Loon, J. Th. \& Oliveira, J. M. 2003, A\&A, in press

Walter, F. M., Wolk, S. J., \& Sherry, W. H. 1998, in The Tenth Cambridge Workshop on Cool Stars, Stellar Systems, and the Sun, R. A. Donahue \& J. A. Bookinder (eds), ASP Conf. Ser. 154, p. 1793

Zapatero Osorio, M. R. et al. 2000, Science, 290, 103

Zapatero Osorio, M. R. et al. 2002, ApJ, 578, 536

This preprint was prepared with the AAS LATEX macros v5.0. 
Fig. 1.- NIRSPEC $J$-band spectra of SOri70 and the T5 field brown dwarf 2MASS J0559191-140449 (Burgasser et al. 2000). Data have been normalized to unity at $1.235-1.24 \mu \mathrm{m}$, and have been shifted for clarity. Note the strong water vapor absorption present in the spectrum of S Ori 70, and the "flat" spectrum of the field brown dwarf. Major molecular and atomic features are indicated.

Fig. 2.- COND synthetic spectra computed for $T_{\text {eff }}=1000 \mathrm{~K}$ and two different gravities: $\log g=3.5$ and $5.5\left(\mathrm{~cm} \mathrm{~s}^{-2}\right)$. These spectra were kindly provided by France Allard and are described in Allard et al. (2001). At these wavelengths, the low-gravity model shows stronger water vapor absorption and stronger $\mathrm{K}$ I lines than does the high-gravity model. Theoretical spectra have been degraded to the resolution of NIRSPEC data (about $8 \AA$ ).

Fig. 3.- The NIRSPEC spectrum of S Ori 70 (solid line) compared with the COND synthetic spectrum with $T_{\text {eff }}=1100 \mathrm{~K}$ and $\log g=3.5 \mathrm{~cm} \mathrm{~s}^{-2}$. As in Fig. 1, major molecular and atomic features are indicated.

Fig. 4.- Location of S Ori 70 (filled circle) in the gravity versus effective temperature diagram. Evolutionary models are taken from Burrows et al. (1997). From top to bottom, tracks for brown dwarf and planetary masses are delineated with solid lines (masses are labelled in units of Jupiter). The 3 and $10 \mathrm{Myr}$ isochrones are plotted with dotted lines. Also shown with open circles are the locations of the T-class field brown dwarfs G1229 B and Gl570 D (Allard et al. 1996; Geballe et al. 2001). From the figure, we derive that S Ori 70 is younger than $10 \mathrm{Myr}$, with a likely age of $3 \mathrm{Myr}$, and has a planetary mass of $3_{-2}^{+5}$ times the mass of Jupiter. 


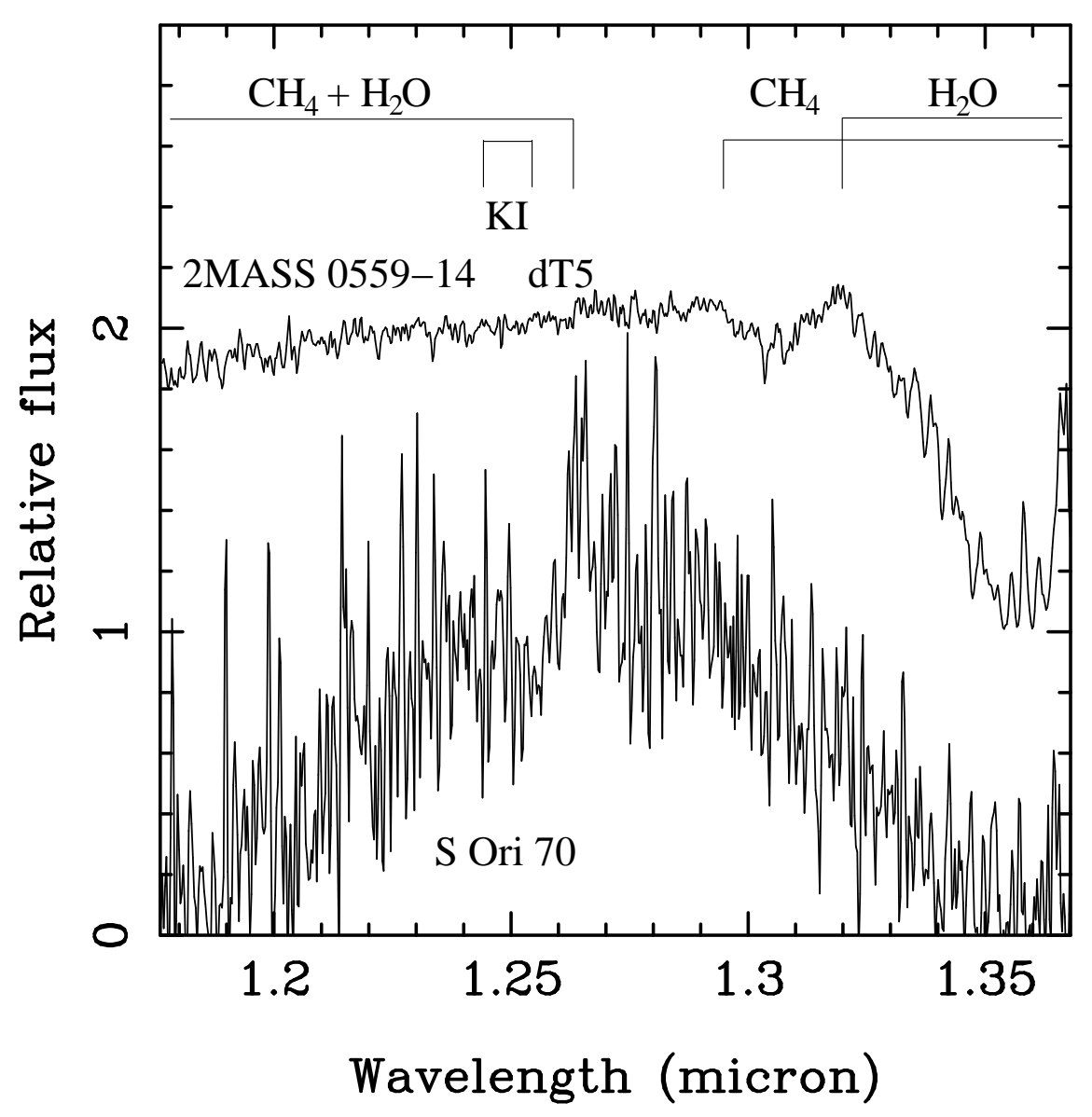




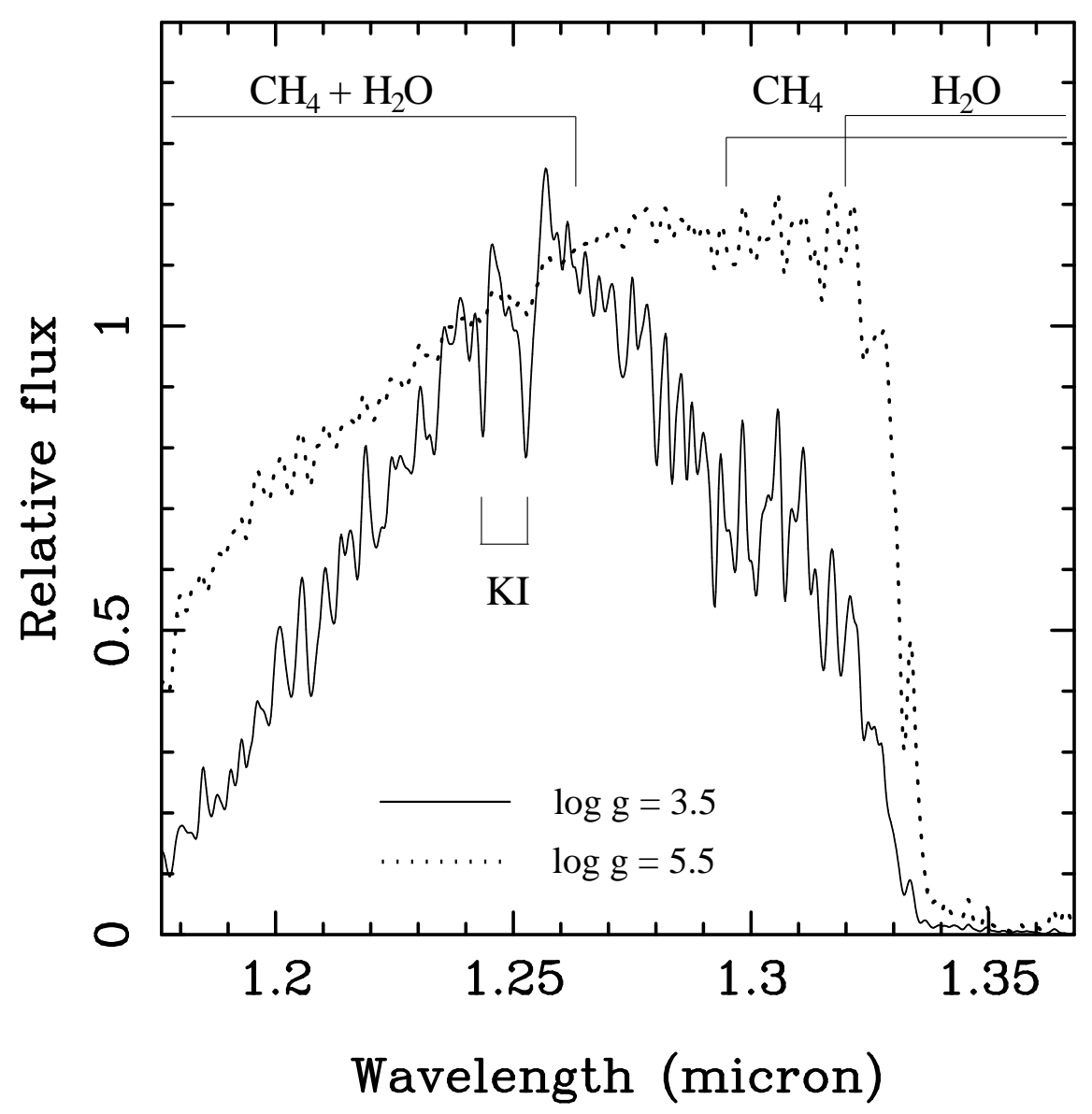




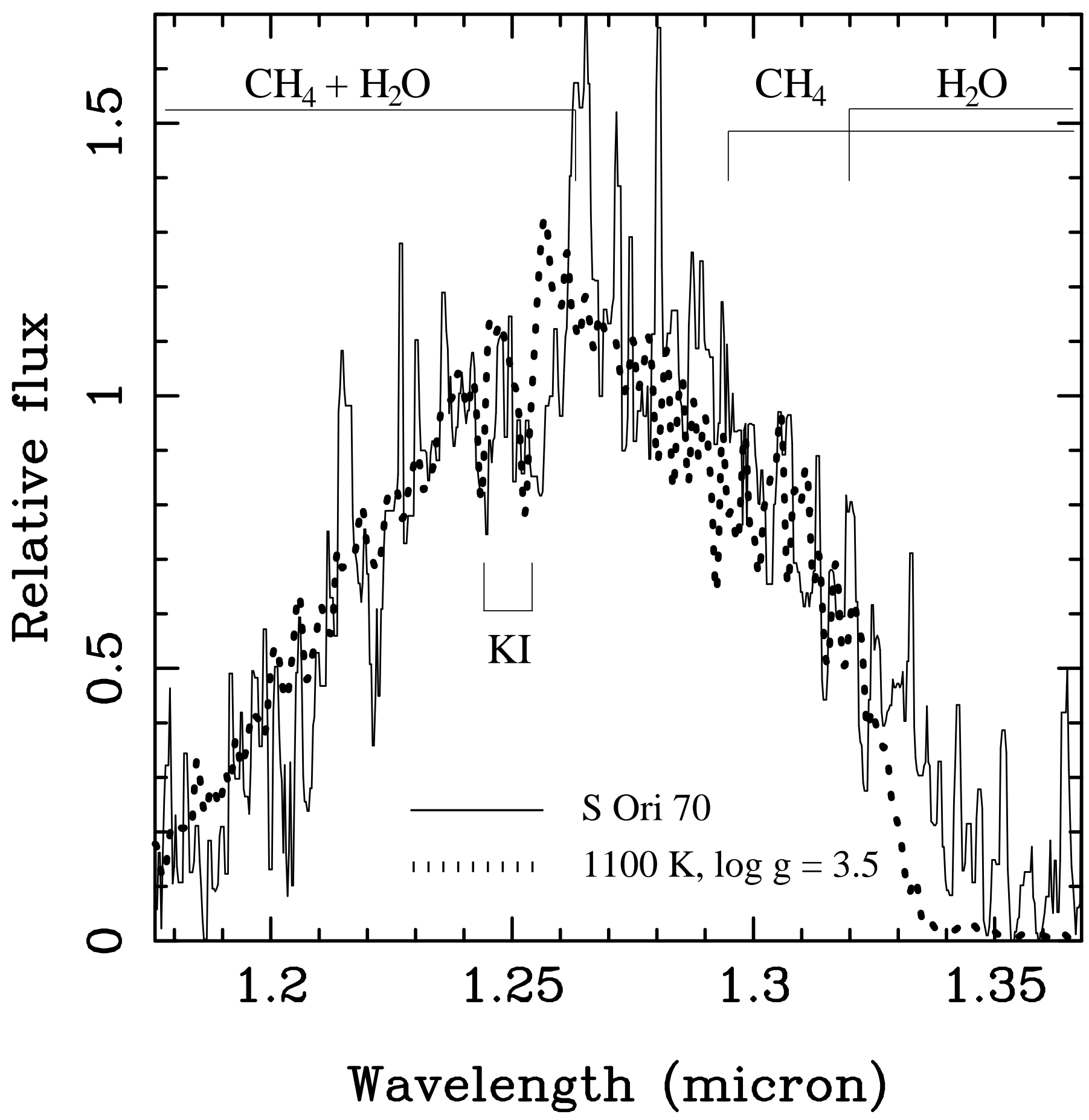




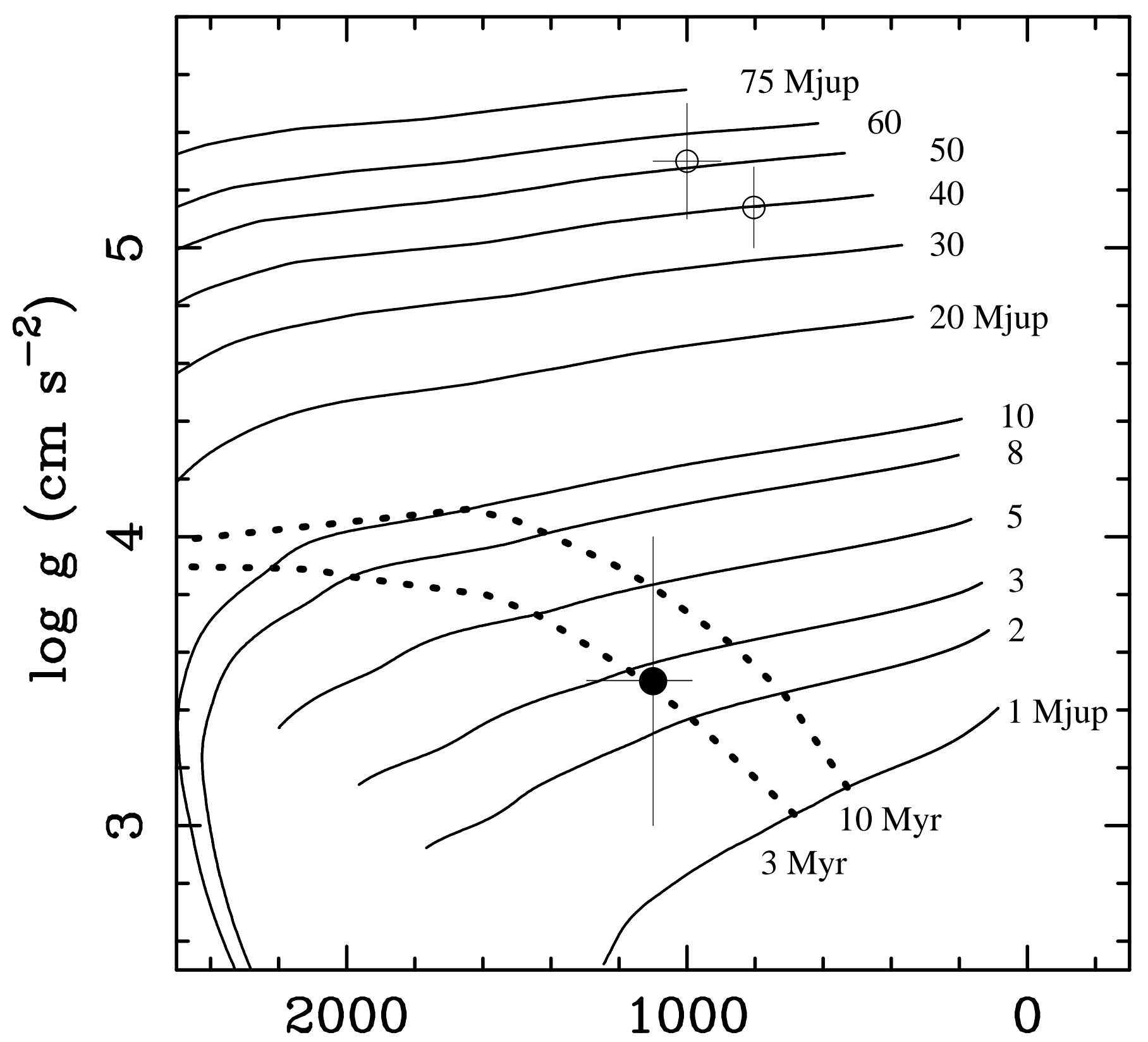

Teff (K) 